\title{
Is there evidence for post-translational modification of beta cell autoantigens in the aetiology and pathogenesis of type 1 diabetes?
}

\author{
Åke Lernmark \\ Received: 11 July 2013 / Accepted: 8 August 2013 /Published online: 11 September 2013 \\ (C) Springer-Verlag Berlin Heidelberg 2013
}

\begin{abstract}
Størling and colleagues hypothesise in this issue (Diabetologia DOI:10.1007/s00125-013-3045-3) that posttranslational modification (PTM) of autoantigens might create tissue-specific neo-epitopes that could trigger type 1 diabetes. Data on PTM of islet autoantigens are scarce and readers should not believe that the PTM hypothesis is supported by strong experimental evidence. The proposed genetic factors are many but their possible contribution is conjectural. There is a lack of a rational approach to test the PTM hypothesis at the different stages of type 1 diabetes. Research that carefully addresses each stage of the type 1 diabetes disease process is warranted to advance our understanding of autoimmune (type 1) diabetes.
\end{abstract}

Keywords Antigen presentation · Antigen processing · Autoantigens · Autoimmune diabetes · Cellular immunity · Glutamic acid decarboxylase $\cdot$ HLA-DQ $\cdot$ Humoral immunity $\cdot$ IA-2 $\cdot$ Insulin $\cdot$ ZnT8

\section{Abbreviations \\ APC Antigen presenting cells \\ PTM Post-translational modification \\ ZnT8 Zinc transporter 8}

Autoimmune (type 1) diabetes mellitus is a multi-stage disease. Our current understanding of the aetiology and pathogenesis of the disease is incomplete and fragmentary. It is incomplete because the mechanisms that trigger the autoimmune reaction against the beta cells are still essentially

\footnotetext{
Å. Lernmark $(\bowtie)$

Department of Clinical Sciences, Lund University/CRC, Skåne

University Hospital SUS, Jan Waldenströms gata 35,

SE-205 02 Malmö, Sweden

e-mail: ake.lernmark@med.lu.se
}

unknown. It is fragmentary because observations related to autoimmune phenomena are typically based on investigations conducted at the time of clinical onset of the disease or, at best, in individuals who have developed persistent islet autoantibodies. In 1986, George Eisenbarth grouped the development of autoimmune (type 1) diabetes into the following stages [1]: (1) genetic susceptibility; (2) a hypothetical triggering event (perhaps a virus) resulting in (3) islet autoimmunity associated with loss of beta cell function; and (4) clinical onset of diabetes. The final stage would be caused by a combination of a deficiency in insulin production and variable insulin sensitivity. In the accompanying For Debate article it is hypothesised that post-translational modification (PTM) of autoantigens might create tissue-specific neo-epitopes that could trigger type 1 diabetes [2]. It should be noted that the contribution by Størling et al [2] truly fulfils the criterion for a 'For Debate' article in that the authors do not provide strong experimental evidence for PTM. Before discussing whether this hypothesis is testable or not, it will first be important to briefly examine the multi-stage character of autoimmune (type 1) diabetes.

Up until recently, stage 1 studies were based on individuals who had already reached and passed stage 4, i.e. they had diabetes. This was a necessary proxy of individuals at risk for stage 4-clinical onset of autoimmune (type 1) diabetes. A weakness was that genetic factors exclusively important to stages 2 and 3 might have been missed. Current information on so-called type 1 diabetes genes are somewhat complicated by the fact that the bulk of genetic information is from studies of first-degree relatives [3]. In most countries, only $10-13 \%$ of newly diagnosed type 1 diabetic patients have a first-degree relative with the disease.

Studies of stage 2 are scant as children with increased genetic risk need to be followed from birth, perhaps at weekly but definitely at monthly intervals. Currently, there is no marker of a trigger event that results in islet autoimmunity. It 
is taken for granted but needs to be proven that the appearance of an islet autoantibody is preceded by cellular events involving antigen-presenting cells (such as dendritic cells or macrophages), $\mathrm{CD}^{+} \mathrm{T}$ cells and $\mathrm{B}$ cells. Current islet autoimmunity tests measure levels of IgG1, IgG2 and IgG4 but not IgG3 or $\operatorname{IgM}$ (which usually precede $\operatorname{IgG}$ ). Other isotypes such as IgA or IgE are not determined. Dissecting the stage 2 triggering event remains an extraordinary challenge.

Clinical studies of stage 3 are possible with some accuracy since the IgG islet autoantibody tests have been subject to international standardisation and harmonisation [4-6]. Autoantibodies against insulin have been studied since 1983 [7] and following the development of a radiobinding autoantibody assay [8], tests for GAD65, IA-2 [4] and, more recently, for zinc transporter 8 (ZnT8) [6] have also been harmonised. The major efforts to dissect stage 3 and find ways not only to predict [9] but also prevent $[10,11]$ the clinical diagnosis have largely been dependent on these harmonised tests [5]. For example, a recent risk score exercise by the TrialNet Study group suggested that levels of multiple autoantibodies may be included in an autoantibody risk score that accurately predicts type 1 diabetes in relatives [9]. Assays that predict progression to clinical onset better than the islet autoantibodies are yet to be developed; these could potentially be for components of antigen presenting cells (APC), T cells or B cells $[12,13]$.

Stage 2 would represent a short-lasting (days to weeks) immune response to a beta cell insult, perhaps a virus infection [14]. Molecular mimicry could be important to stage 2 [15]. Stage 3, on the other hand, is different in terms of time scale (months to years) and scope. The century-old notion that a virus is the trigger of type 1 diabetes, first proposed because infections were often recorded close to stage 4 , has been misleading. Nowadays it is possible to hypothesise that virus infections during stage 3 might accelerate chronic islet autoimmunity [16].

Is it possible that PTM plays a prominent role in triggering beta cell destruction? The authors do not provide strong experimental evidence for PTM of autoantigens. Therefore, the question needs to be answered by examining each of stages $1-3$. Is it possible that stage 1 has something to do with a process involving PTM? This question is best answered once it has been determined whether there are genetic factors important to the induction of stage 3 islet autoimmunity, which, at present, means IgG autoantibodies against islet autoantigens. Whether these genes code for enzymes important to PTM remains to be determined. It may also be necessary to consider genetic factors that are more important to the immediate immune response of stage 2 rather than the more chronic islet autoimmunity of stage 3 .

Størling et al [2] propose that increased expression levels (mRNA, not the enzymes themselves) of enzymes with potential PTM activity after in vitro exposure of human islets to certain cytokines indicates that PTM of autoantigens might take place. Regrettably, there are quite a number of candidate PTM enzymes to sort through. However, it is a testable hypothesis to use these enzymes to explore whether insulin, GAD65, IA-2 or ZnT8 are substrates for PTM reactions. Palmitoylation of GAD65 seemed to control cellular trafficking but not the recognition of autoantibodies [17]. The primary outcome should not be altered reactivity with the $\mathrm{IgG}$ islet autoantibodies, but, rather, processing and presentation by APC on HLA DR-DQ Class II heterodimers and the subsequent responses by $\mathrm{CD} 4^{+} \mathrm{T}$ cells and, possibly, B cells.

In the event that stage 2 involves the HLA-DQ heterodimers, it will be of particular importance to dissect whether the response involves all four heterodimers that can be generated between DQ A1*03:01-B1*03:02 (DQ8) and $\mathrm{A} 1 * 05: 01-\mathrm{B} 1 * 02: 01$ (DQ2). It needs also to be explained why the subsequent (several steps later) formation of $\operatorname{IgG}$ islet autoantibodies are associated with HLA-DQ. Autoantibodies to insulin and IA-2 have been found to be associated with DQ8, GAD65 autoantibodies with DQ2 [18] and ZnT8 autoantibodies with DQ6.4 [19]. Autoantibodies to IA-2 and ZnT8 were negatively associated with DQ2 [18, 19]. A major question not asked by Størling et al is whether the loading of peptides in the binding cleft of the HLA Class II heterodimer is affected by PTMs [2]. Are post-translationally modified peptides loaded at all? If not, $\mathrm{T}$ cells with $\mathrm{T}$ cell receptors (TCRs) that recognise an autoantigen peptide may escape negative selection and migrate to the periphery to increase subsequent risk for autoimmunity. The mechanisms by which PTM-modified autoantigens could contribute may therefore be the other way around than that proposed by Størling et al [2].

Recent studies suggest that IgG islet autoantibodies may be present in children as young as 1-3 years of age [20]. Once an IgG autoantibody is detectable, it may be a reasonable assumption that autoantigen presentation took place at least a month before. Størling et al speculate that the triggering event prior to antigen presentation may involve PTM of autoantigens [2]. This hypothesis is weakened by observations that seroconversion is sequential and that the different islet autoantibodies do not appear at the same time [20]. Research needs to be focused on stage 2, which, to some extent, is what both the Type 1 Diabetes Prediction and Prevention Project (DIPP) [14] and The Environmental Determinants of Diabetes in the Young (TEDDY) [21] studies have done to answer the question of whether a triggering event, such as an enterovirus infection of beta cells, would be associated with an increase (or decrease) in PTM autoantigens.

Would PTM of autoantigens be important to stage 3? A very important characteristic of stage 3 is the variable length of time of progression to clinical onset. Children may develop two or more IgG islet autoantibodies at 1-3 years of age. Some of them may develop diabetes, i.e complete stage 3 within 6 months [22]. In other children with two or more 
IgG islet autoantibodies, progression to diabetes may take years [20]. It has been speculated that this variation could be due to non-HLA genetic factors that would either decelerate or accelerate the pathogenic process. With time, the child may progress from one to two and even to three or to four IgG islet autoantibodies. This increase in the number of different autoantibodies would coincide with chronic beta cell autoimmunity. A reasonable explanation for this chronic state would be that beta cell killing takes place, perhaps at an increasing rate, by autoantigen-specific $\mathrm{CD} 8^{+} \mathrm{T}$ cells [23]. This process is highly beta cell specific because only short peptides from intracellular proteins such as insulin, GAD65, IA-2 and ZnT8 are presented on the HLA class I proteins on the beta cell surface. Studies of human pancreas from IgG islet autoantibody-positive individuals suggest an increased expression of HLA class I proteins on the beta cells. The risk of beta cells being detected by $\mathrm{CD} 8^{+} \mathrm{T}$ cells recognising autoantigen peptides is increased. The mechanism by which beta cells are specifically eradicated is through autoantigen peptides presented on HLA class I proteins. The suggestion by Størling et al [2] that only insulin, but not GAD65, IA- 2 and $\mathrm{ZnT}$, is beta cell specific is therefore irrelevant.

A more relevant question is: are the peptides presented on HLA class I on the beta cell surface altered by PTM of the autoantigen? This would imply that there are PTM processes during stage 3 that would enhance the interaction between beta cell HLA class I proteins and the T cell receptors on $\mathrm{CD}^{+}$ $\mathrm{T}$ cells. Autoantigens modified by PMT before being processed into peptides and loaded on the class I heterodimer may also represent a neo-antigen. This could be the way that virus infections that occur during stage 3 accelerate progression to clinical onset [16]. If this were the case, the involvement of PTMs, if any, would be to give rise to changed peptide structures creating neo-epitopes during the chronic, escalating islet autoimmunity during stage 3 . During this stage, the beta cells are under an increasing escalating attack by $\mathrm{CD} 8^{+} \mathrm{T}$ cells. Killed beta cells are likely to be processed by APCs to allow epitope spreading to new epitopes on the same autoantigen or to other autoantigens (antigen spreading). T cell assays will be needed to test this hypothesis as islet autoantibodies, although they may change in terms of titre and epitope specificity [9], are too far removed from the actions of $\mathrm{CD} 8^{+} \mathrm{T}$ cells.

In summary, the question of whether PTM of beta cell proteins trigger type 1 diabetes is difficult to answer. It is important that the reader should not be misled to believe that the PTM hypothesis is supported by strong experimental evidence. Data on PTM of islet autoantigens are scarce. The proposed genetic factors could be non-causal polymorphic traits. There is a lack of a rational approach to test the PTM hypothesis at the different stages of type 1 diabetes. One hope is that the study by Størling et al will trigger a burst of research activities that carefully address each stage of the disease process, and generate robust, reproducible data. Regardless of whether this supports or refutes the PTM hypothesis, it will advance our understanding of autoimmune (type 1) diabetes.

Funding The research in the author's laboratory is supported by the National Institutes of Health (DK63861), JDRF, Swedish Research Council, Swedish Diabetesfund, SUS Funds and the Skåne County Council for Research and Development.

Duality of interest The author is a member of the Scientific Advisory Boards of Diamyd Medical AB, Stockholm, Sweden, Probi AB, Lund, Sweden and Zealand Pharma A/S, Copenhagen, Denmark.

Contribution statement The author was the sole contributor to this paper.

\section{References}

1. Eisenbarth GS (1986) Type I diabetes mellitus. A chronic autoimmune disease. N Engl J Med 314:1360-1368

2. Størling J, Overgaard AJ, Brorsson CA et al (2013) Do posttranslational beta cell protein modifications trigger type 1 diabetes? Diabetologia doi:10.1007/s00125-013-3045-3

3. Concannon P, Rich SS, Nepom GT (2009) Genetics of type 1A diabetes. N Engl J Med 360:1646-1654

4. Torn C, Mueller PW, Schlosser M, Bonifacio E, Bingley PJ (2008) Diabetes Antibody Standardization Program: evaluation of assays for autoantibodies to glutamic acid decarboxylase and islet antigen-2. Diabetologia 51:846-852

5. Bonifacio E, Yu L, Williams AK et al (2010) Harmonization of glutamic acid decarboxylase and islet antigen-2 autoantibody assays for national institute of diabetes and digestive and kidney diseases consortia. J Clin Endocrinol Metab 95:3360-3367

6. Lampasona V, Schlosser M, Mueller PW et al (2011) Diabetes antibody standardization program: first proficiency evaluation of assays for autoantibodies to zinc transporter 8. Clin Chem 57: 1693-1702

7. Palmer JP, Asplin CM, Clemons P et al (1983) Insulin antibodies in insulin-dependent diabetics before insulin treatment. Science 222: $1337-1339$

8. Grubin CE, Daniels T, Toivola B et al (1994) A novel radioligand binding assay to determine diagnostic accuracy of isoform-specific glutamic acid decarboxylase antibodies in childhood IDDM. Diabetologia 37:344-350

9. Sosenko JM, Skyler JS, Palmer JP et al (2013) The prediction of type 1 diabetes by multiple autoantibody levels and their incorporation into an autoantibody risk score in relatives of type 1 diabetic patients. Diabetes Care 36:2615-2620

10. Diabetes Prevention Trial-Type 1 Diabetes Study Group (2002) Effects of insulin in relatives of patients with type 1 diabetes mellitus. N Engl J Med 346:1685-1691

11. Gale EA, Bingley PJ, Emmett CL, Collier T (2004) European Nicotinamide Diabetes Intervention Trial (ENDIT): a randomised controlled trial of intervention before the onset of type 1 diabetes. Lancet 363:925-931

12. James EA, Mallone R, Schloot NC et al (2011) Immunology of Diabetes Society T-Cell Workshop: HLA class II tetramer-directed epitope validation initiative. Diabetes Metab Res Rev 27:727-736

13. Brooks-Worrell B, Tree T, Mannering SI et al (2011) Comparison of cryopreservation methods on T-cell responses to islet and control antigens from type 1 diabetic patients and controls. Diabetes Metab Res Rev 27:737-745 
14. Oikarinen S, Martiskainen M, Tauriainen S et al (2011) Enterovirus RNA in blood is linked to the development of type 1 diabetes. Diabetes 60:276-279

15. Masala S, Paccagnini D, Cossu D et al (2011) Antibodies recognizing Mycobacterium avium paratuberculosis epitopes cross-react with the beta-cell antigen ZnT8 in Sardinian type 1 diabetic patients. PLoS One 6:e26931

16. Stene LC, Oikarinen S, Hyoty H et al (2010) Enterovirus infection and progression from islet autoimmunity to type 1 diabetes: the Diabetes and Autoimmunity Study in the Young (DAISY). Diabetes 59:3174-3180

17. Baekkeskov S, Kanaani J (2009) Palmitoylation cycles and regulation of protein function (review). Mol Membr Biol 26: $42-54$

18. Graham J, Hagopian WA, Kockum I et al (2002) Genetic effects on age-dependent onset and islet cell autoantibody markers in type 1 diabetes. Diabetes 51:1346-1355
19. Delli AJ, Vaziri-Sani F, Lindblad B et al (2012) Zinc transporter 8 autoantibodies and their association with SLC30A8 and HLA-DQ genes differ between immigrant and Swedish patients with newly diagnosed type 1 diabetes in the Better Diabetes Diagnosis study. Diabetes 61:2556-2564

20. Ziegler AG, Rewers M, Simell O et al (2013) Seroconversion to multiple islet autoantibodies and risk of progression to diabetes in children. JAMA 309:2473-2479

21. Hagopian WA, Erlich H, Lernmark A et al (2011) The Environmental Determinants of Diabetes in the Young (TEDDY): genetic criteria and international diabetes risk screening of 421000 infants. Pediatr Diabetes 12:733-743

22. Lee HS, Briese T, Winkler C et al (2013) Next-generation sequencing for viruses in children with rapid-onset type 1 diabetes. Diabetologia 56:1705-1711

23. Roep BO, Peakman M (2012) Antigen targets of type 1 diabetes autoimmunity. Cold Spring Harb Perspect Med 2:a007781 